\title{
Locked-in Syndrome Due to Meningovascular Syphilis: A Case Report and Literature Review
}

\author{
Yuki Yokota ${ }^{1}$, Masaki Ishihara ${ }^{1}$, Satoko Ninomiya ${ }^{1}$, Kazutaka Mitsuke $^{1}$, \\ Satoshi Kamei $^{2}$ and Hideto Nakajima ${ }^{1}$
}

\begin{abstract}
:
We herein report a 46-year-old man presenting with locked-in syndrome secondary to meningovascular syphilis. Brain magnetic resonance imaging (MRI) demonstrated multiple acute infarctions in the left ventromedial pons, right basis pontis, and left basal ganglia. His locked-in syndrome was hypothesized to have been caused by thrombosis of the small paramedian branches of the basilar artery due to syphilitic arteritis. This is a unique case of bilateral ventromedial pontine infarction caused by meningovascular syphilis that presented as locked-in syndrome. Meningovascular syphilis should be included in the differential diagnosis of uncommon stroke, particularly in young men.
\end{abstract}

Key words: neurosyphilis, meningovascular syphilis, brainstem infarction, locked-in syndrome, young adult, uncommon stroke

(Intern Med Advance Publication)

(DOI: 10.2169/internalmedicine.8269-21)

\section{Introduction}

Neurosyphilis refers to infection of the central nervous system (CNS) by the spirochete Treponema pallidum, and it occurs within the first year after infection, even though CNS involvement is possible at any stage of the disease (1-4). While long-term sequelae of the disease include dementia paralytica and tabes dorsalis, early neurosyphilis can cause meningitis, gumma, and stroke due to meningeal vasculopathy.

Locked-in syndrome is a rare neurological condition characterized by quadriplegia and mutism but with a preserved consciousness $(5,6)$ wherein patients can sometimes communicate through eye movements or blinking. This catastrophic disorder is typically caused by bilateral infarction of the ventromedial pontine.

We herein report a man with locked-in syndrome due to meningovascular syphilis who was human immunodeficiency virus (HIV)-negative.

\section{Case Report}

A 46-year-old left-handed Japanese man was admitted to another hospital for acute-onset right-sided spastic hemiparesis. His recent history included mild fatigue, pyrexia, and severe headaches accompanied by exacerbation of nausea and anorexia for the preceding three weeks. He was transferred to our hospital two days after the onset of right hemiparesis due to increasing muscle weakness, progressive and severe dysarthria, and dysphagia.

On admission, he was slightly febrile (temperature, $37.1^{\circ} \mathrm{C}$ ), blood pressure was high at $200 / 128 \mathrm{mmHg}$, and pulse was regular at $61 \mathrm{bpm}$. He had a smoking habit (15 cigarettes/day for 29 years), and his medical history included gastric ulcer and alcoholism; however, there was no history of hypertension, diabetes, dyslipidemia, atrial fibrillation, or heart disease.

A physical examination revealed the presence of welldemarcated erythematous papules and plaques with scaling on the trunk, the abdomen, and the extremities. On a neurological examination, he was awake, alert, and able to com-

${ }^{1}$ Department of Neurology, Nihon University School of Medicine, Graduate School of Medicine, Japan and ${ }^{2}$ Department of Neurology, Center for Neuro-infection, Ageo Central General Hospital, Japan

Received: July 5, 2021; Accepted: September 1, 2021; Advance Publication by J-STAGE: October 19, 2021

Correspondence to Dr. Masaki Ishihara, ishihara.masaki@nihon-u.ac.jp 

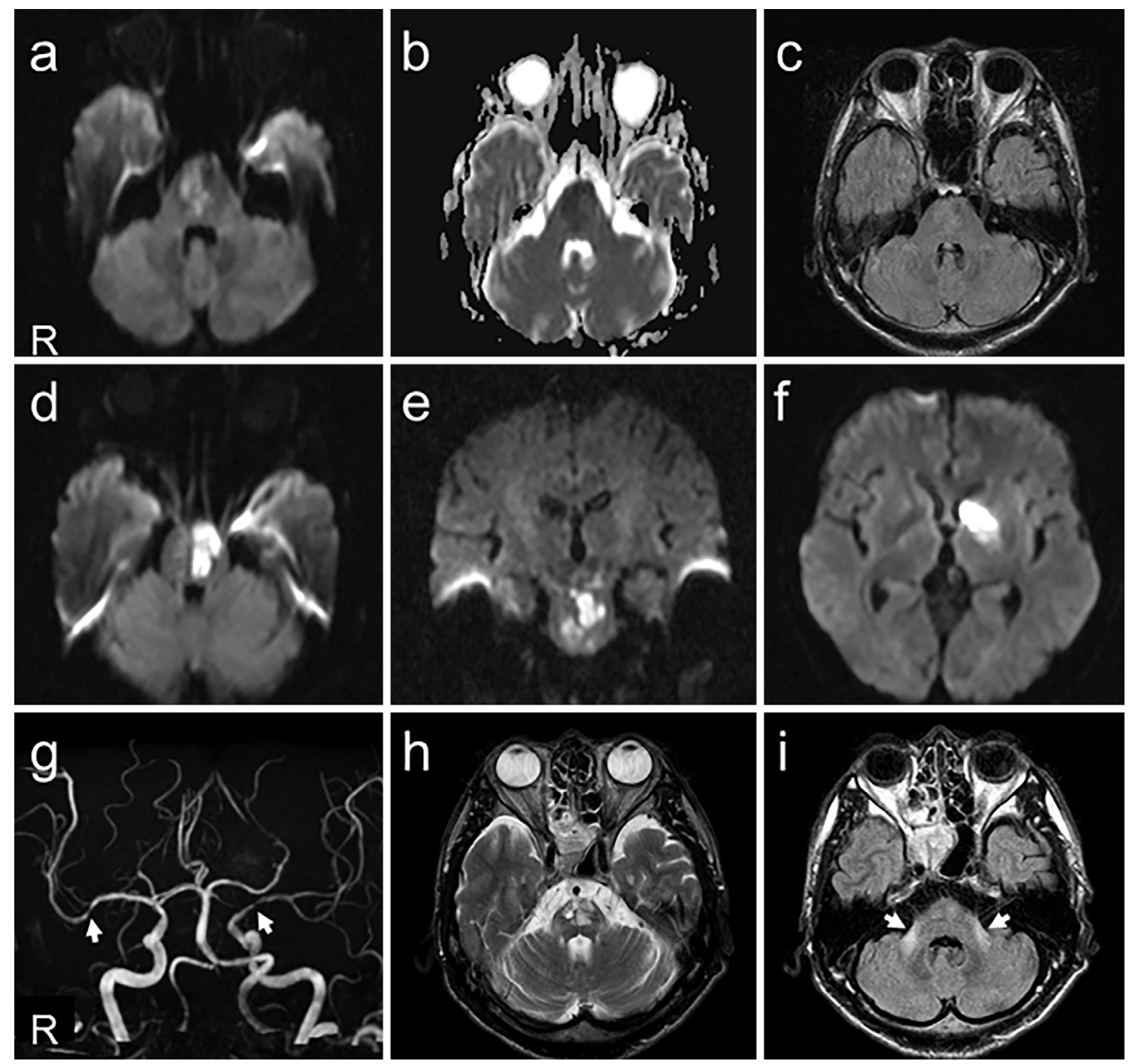

Figure 1. Brain MRI and MRA findings. On the day of admission, brain MRI showed high-intensity lesion in the left ventromedial pons and slight high-intensity lesion in the right ventromedial pons on diffusion-weighted imaging (DWI) (a, d, e), with low-intensity findings noted on an apparent diffusion coefficient (ADC) map (b), high-intensity findings on fluid-attenuated inversion recovery (FLAIR) imaging (c), and high-intensity findings in the left basal ganglia, including the caudate nucleus on DWI (f). MRA showed narrowing of the bilateral middle cerebral artery (MCA) (g, arrows). Eight months after the onset of infarction, follow-up brain MRI showed an old infarction in the bilateral ventromedial pons on T2-weighted imaging (h). High-intensity lesions in the bilateral middle cerebellar peduncles, where the pontocerebellar tract runs, suggested Wallerian degeneration secondary to a pontine infarction (FLAIR) (i, arrows).

municate with a nodding motion but unable to speak. He displayed quadriplegia with preserved full eye movement and blinking, and the muscle strength in his extremities was $1 / 5$. Hyperreflexia was present in all extremities along with bilateral positive Babinski's sign. At best, sensory testing is difficult to perform accurately, and on day 11 of hospitalization, his neurological condition worsened significantly, with only consciousness, breathing, eye movement in all directions, and blinking preserved; no movements in the limbs, mouth, or tongue, including swallowing movements, were observed. Therefore, a nasogastric tube was inserted to deliver nutrients to the patient.

Brain magnetic resonance imaging (MRI) on admission day revealed a high-intensity lesion in the left ventromedial pons, along with a small high-intensity lesion in the right basis pontis and in the left basal ganglia, including in the caudate nucleus, on diffusion-weighted imaging (DWI) (Fig. 1a, d-f). Considering the apparent diffusion coefficient map and fluid-attenuated inversion recovery (FLAIR) imaging, these findings were consistent with acute cerebral infarctions. Magnetic resonance angiography (MRA) showed a normal basilar artery flow but bilateral partial stenosis of the middle cerebral artery (MCA) (Fig. 1e). Brain MRI acquired after neurological deterioration revealed a high-intensity lesion in the right basis pontis. Contrast-enhanced MRI showed no abnormal findings specific to meningovascular 


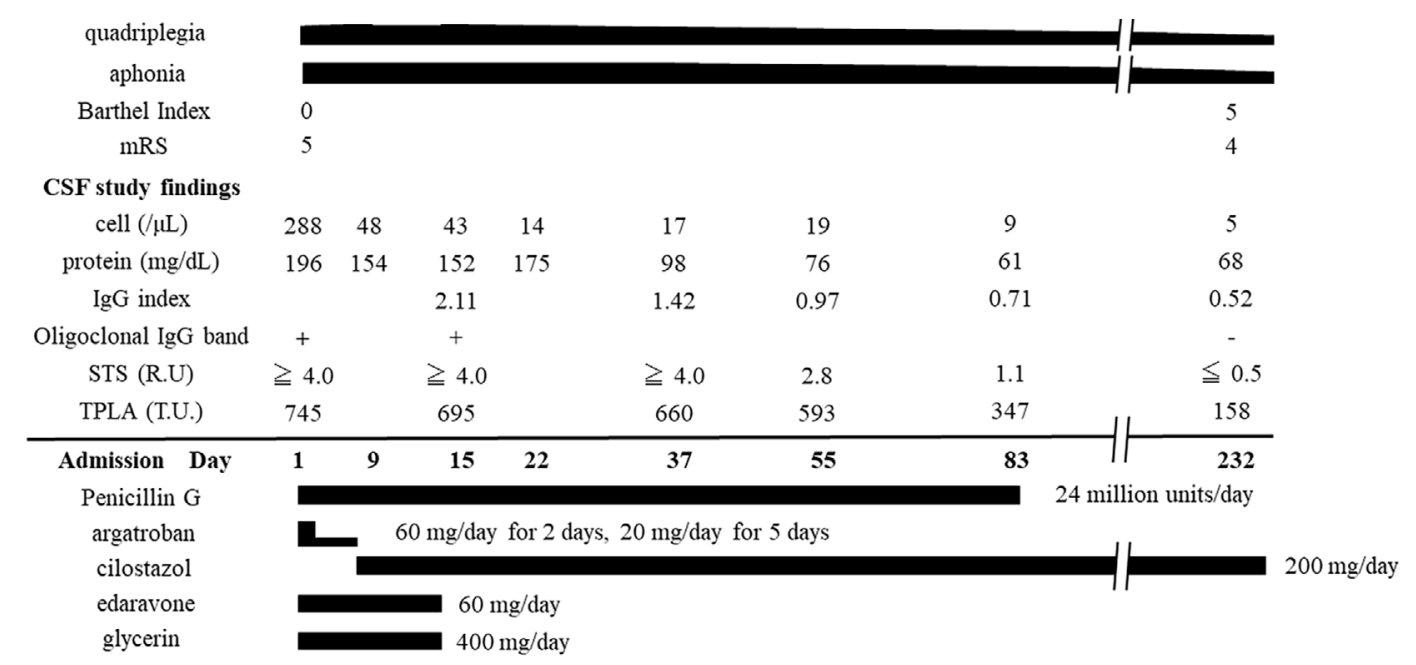

Figure 2. Clinical course of the patient. mRS: modified Rankin Scale, CSF: cerebrospinal fluid, STS: serological test for syphilis, TPLA: Treponema pallidum latex agglutination

syphilis, except for findings consistent with the natural course of cerebral infarction in the left basal ganglia. MRA showed a normal basilar artery flow similar to the previous findings. Consciousness was clear during the clinical course. Although an electroencephalogram test was performed, it was not useful for evaluating the background activity because of the inability to exclude artifacts. An electrocardiogram and chest $\mathrm{X}$-ray findings were unremarkable, and a serum test for HIV antibodies was negative. Screening for hepatitis B virus (HBV) serology displayed the following pattern: hepatitis B surface antigen (HBsAg)-negative, antihepatitis B surface antibody (anti-HBsAb)-positive, antihepatitis B core antibody (anti-HBcAb)-positive, and HBV-DNA not detectable by real-time detection polymerase chain reaction.

Routine laboratory evaluations revealed an elevated white blood cell count $(17,900 / \mu \mathrm{L}$; neutrophil $88.8 \%)$ and Creactive protein levels $(0.80 \mathrm{mg} / \mathrm{dL})$. A serological test for syphilis (STS; cut-off point: 1 RPR units [R.U.]) and T. pallidum latex agglutination (TPLA; cut-off point: 10 titer units [T.U.]) test were positive at 64 R.U. and 14,347 T.U., respectively. A cerebrospinal fluid (CSF) analysis was remarkable for the presence of cells $(288 / \mu \mathrm{L})$, mainly lymphocytes, with high levels of protein $(196 \mathrm{mg} / \mathrm{dL})$, glucose $(30 \mathrm{mg}$ / dL), STS (8 R.U., cut-off: 1 R.U.), and TPLA (745 T.U., cut-off: 10 T.U.). Both serum and the CSF were positive for the fluorescent treponemal antibody-absorption test. An elevated CSF IgG index $(2.11$; cut-off $<0.7)$ with positive oligoclonal IgG bands (OCB) was also detected, suggesting intrathecal production of anti-treponemal antibodies. Polymerase chain reaction for herpes simplex virus and varicella zoster virus was negative, and no findings indicative of cardiogenic cerebral embolism were present. Based on the above, the patient was diagnosed with meningovascular syphilis, and Fig. 2 shows the clinical course of the patient.

He was treated with intravenous penicillin at a dose of 4 million units, 6 times a day, for 12 weeks and was pre- scribed intravenous argatroban, edaravone, and glycerin for cerebral infarction, followed by oral cilostazol tablets. We did not administer steroids due to concerns of deep-vein thrombosis complications and $\mathrm{HBV}$ reactivation. Both the IgG index and OCB normalized gradually and in parallel with improvements in CSF parameters.

Four months after the onset, he required full assistance for his daily activities, and he was transferred to the Department of Rehabilitation Medicine after penicillin therapy. During follow-up at 8 months after the diagnosis and treatment for neurosyphilis, the CSF parameters, including CSF IgG index (0.52), had reverted to normal values, except for a slight elevation of the protein concentration. Follow-up brain MRI was remarkable for old infarctions in the bilateral ventromedial pons on T2-weighted imaging (Fig. 1h) and bilateral symmetrical hyperintense lesions at the middle cerebellar peduncles, which suggested Wallerian degeneration secondary to ventromedial pontine infarction (Fig. 1i) $(7,8)$. No abnormal signals were detected in the medulla oblongata or corticospinal tract. During the course, he did not need gastrostomy or mechanical ventilatory support. Intensive rehabilitation slightly improved his Barthel index score from 0 to 5, and although he was able to pronounce vowels, he could not speak in sentences. The muscle strength in the right upper extremity improved gradually, such that he was able to hold a spoon with a self-help device and managed to bring prepared soft food to his mouth. His left upper extremity and lower extremities remained in contracture, and he was unable to independently maintain a seated posture due to brainstem infarction.

\section{Discussion}

We encountered a 46-year-old man with locked-in syndrome due to meningovascular syphilis, which was caused by multiple cerebral infarctions, including brainstem lesions, secondary to symptomatic syphilitic meningitis. The pathol- 
Table. Case Reports of Brainstem Infarction Due to Meningovascular Syphilis.

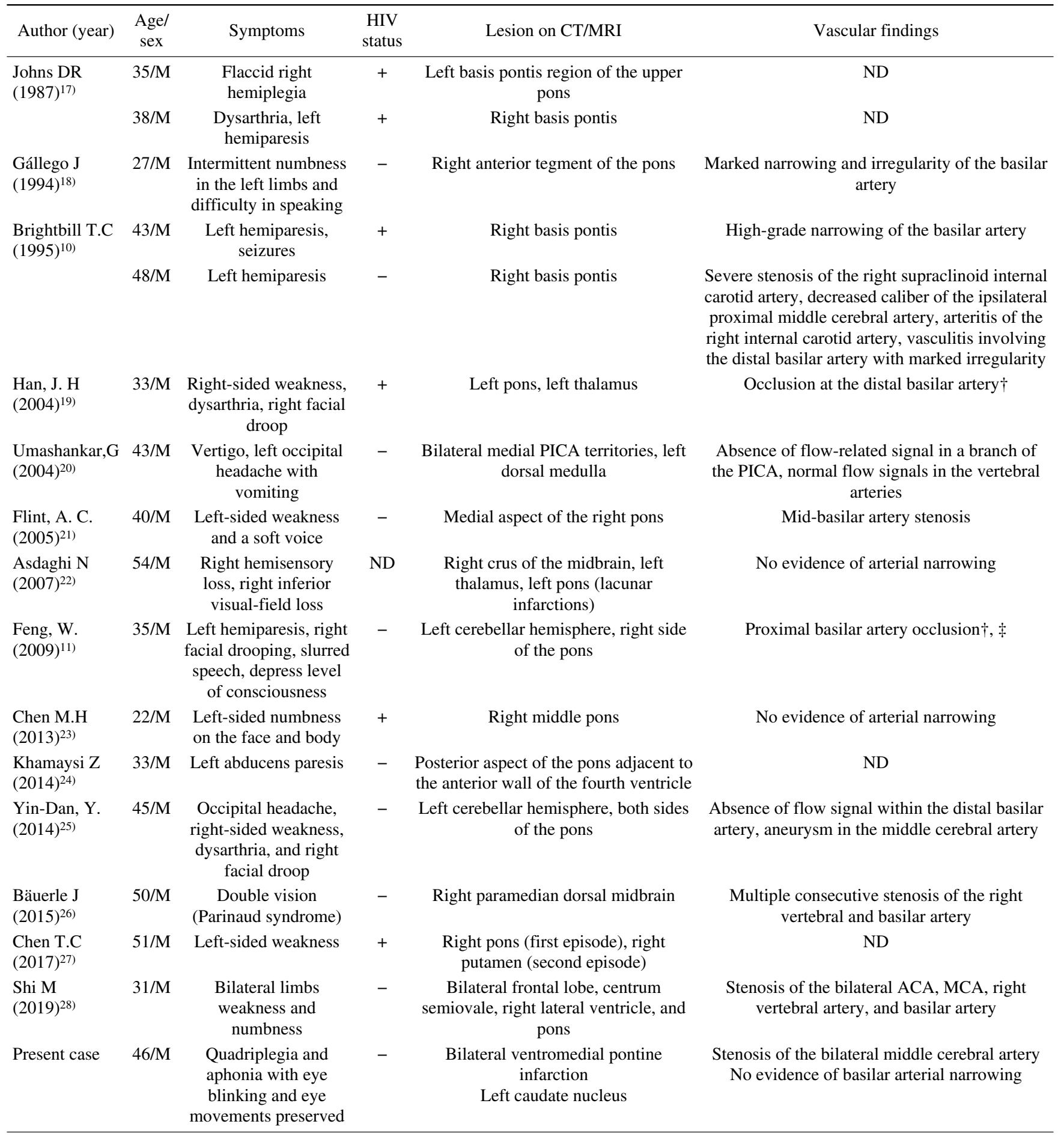

ND: not described, ACA: anterior cerebral artery, MCA: middle cerebral artery

All reported cases revealed pleocytosis, elevated protein concentration, serological test for syphilis, and venereal disease research laboratory in the CSF.

$\dagger$ Tissue-plasminogen activator administration.

†Endovascular recanalization.

ogy underlying meningovascular neurosyphilis is endarteritis of the medium and large arteries (Heubner's arteritis) and of the small arteries and arterioles (Nissl-Alzheimer's arteritis) (9-11), wherein hyperplasia of the subintimal fibrous tissue results in a narrowed lumen that leads to thrombotic occlusion and ischemic infarction (9). The MCA is the most commonly involved artery, followed by the basilar ar- tery (9). In our patient, the basis pontis infarction that led to incomplete locked-in syndrome was assumed to have been caused by thrombosis of small paramedian branches of the basilar artery due to Nissl-Alzheimer-type syphilitic arteritis. In addition, multiple cerebral infractions, including in the left caudate nucleus, implied CNS vasculitis secondary to neurosyphilis. Furthermore, an elevated CSF IgG index with 
OCB pointed toward a specific CNS immune response to the infectious agent (12-15), so the pathogenesis of multiple vascular lesions in this case likely involved both vasculitis and direct invasion by $\mathrm{T}$. pallidum $(3,9-11)$.

A study of imaging findings in neurosyphilis by Brightbill et al. reported that cerebral infarctions were seen in $8(23 \%)$ of 35 patients with neurosyphilis and that brainstem lesions were observed in 2 of 35 cases (10). In contrast, in a report by Peng et al., cerebral infarctions were seen in $6(43 \%)$ of 14 patients, whereas neurosyphilis and brainstem lesions were found in 2 of 14 cases (16). Table shows the details of previous reports of brainstem infarction in meningovascular syphilis $(10,11,17-28)$. The patient age ranged from 22 to 54 years old, all were men, and 6 were HIV-positive. Pontine infarctions were seen in $15(88.2 \%)$ of 17 patients, and some reports have also described the presence of multiple cerebral infarctions, as seen in our case. Only a few patients with acute stroke due to meningovascular syphilis who had occlusion of the vertebrobasilar system were treated with intravenous tissue plasminogen activator and subsequently with antibiotics $(11,19)$.

Locked-in syndrome was first described by Plum and Posner in 1966 (29), and its causes include hemorrhaging, tumor, central pontine myelinolysis, heroin abuse, trauma, pontine abscess, brainstem encephalitis, post-infective polyneuropathy, and air embolism (30). To our knowledge, this is the first report of meningovascular syphilis as the cause of locked-in syndrome. On the ventral side of the pons run the cortical spinal and corticobulbar tracts, a bundle of efferent fibers starting from the motor cortex of the cerebral cortex. The ascending reticular activating system for alert consciousness, named the brain stem reticular formation, has not yet been clarified, but brainstem lesions will not produce coma unless they pass the pontomesencephalic junction in the rostral direction and affect the tegmentum bilaterally $(6,31)$. Brain stem infarction in the territory of the proximal basilar artery bilaterally shows clinical findings of quadriplegia (bilateral cortical spinal tracts), bifacial paralysis, dysarthria, and tongue and mandibular weakness (bilateral corticobulbar tracts) (31). Locked-in syndrome often leads to severe disability or death $(5,32)$, and among cases with the former, the prognosis for non-vascular etiologies, such as trauma, central pontine myelinolysis, tumors, and encephalitis, is better than that for vascular etiologies (5). In addition, a young age, absence of hypertension, and vertebrobasilar insufficiency are considered relatively good prognostic factors (30). Despite our patient's young age and lack of severe stenosis of the basilar artery, he experienced severe sequelae, such as becoming bedridden, despite antiplatelet therapy and extended penicillin treatment, probably because the vascular etiology (i.e. infarctions in the bilateral ventromedial pontine, where the corticospinal and corticobulbar fibers run) led to minimal recovery.

Three options should be considered during treatment of cerebral infarction due to meningovascular syphilis: antibiotics against pathogens (duration of penicillin therapy), stan- dard treatment including antiplatelet therapy for cerebral infarction, and immunotherapy for vasculitis. Penicillin administration for 10-14 days is recommended by the practice guidelines for neurosyphilis in the United States (33), the United Kingdom (34), and Europe (35). As a previous case report indicated that a single course of pharmacotherapy for cerebral infarction and of penicillin failed to prevent relapse of syphilitic vasculitis $(28,36)$, we administered penicillin for 12 weeks until a clear improvement in inflammatory findings and intrathecal antibody production were confirmed. Furthermore, penicillin and antiplatelet treatment alone would be insufficient to improve the prognosis after cerebral infarction. Although we did not add prednisolone to his drug regimen, prednisolone (20-60 mg daily for 3 days) starting the day before anti-treponemal treatment has been recommended in order to prevent syphilitic vasculitis and acute cerebral infarction via the Jarisch-Herxheimer reaction $(3,28)$. Incidentally, a previous report also suggested the need for a second course of penicillin and corticosteroid therapy in syphilis-associated cerebral vasculitis, as despite corticosteroid and antibiotic treatment for one month, relapse occurred after treatment discontinuation (36). However, it should be noted that there is no evidence at present that prophylactic antiplatelet therapy and corticosteroid therapy for infectious cerebral infarction are effective, especially in syphilitic vasculitis $(28,36,37)$. As our patient showed an elevated CSF IgG index with positive OCB, the efficacy of the combination of three approaches for the treatment of cerebral infarction due to meningovascular syphilis should be evaluated.

In summary, we describe a unique cause of bilateral ventromedial pontine infarction, namely meningovascular syphilis, which presented as locked-in syndrome. As brainstem infarction either is fatal or has extremely severe sequelae, meningovascular syphilis should be included in the initial diagnostic workup in young men presenting with uncommon stroke.

The authors state that they have no Conflict of Interest (COI).

\section{Acknowledgements}

The authors would like to thank Enago (www.enago.jp) for the English language review.

\section{References}

1. Ghanem KG, Ram S, Rice PA. The Modern Epidemic of Syphilis. The New England journal of medicine 382: 845-854, 2020.

2. Ropper AH. Neurosyphilis. The New England journal of medicine 381: 1358-63, 2019.

3. Berger JR, Dean D. Neurosyphilis. Handbook of clinical neurology 121: 1461-1472, 2014.

4. Golden MR, Marra CM, Holmes KK. Update on syphilis: resurgence of an old problem. Jama 290: 1510-1514, 2003.

5. Patterson JR, Grabois M. Locked-in syndrome: a review of 139 cases. Stroke 17: 758-764, 1986.

6. Bauer G, Gerstenbrand F, Rumpl E. Varieties of the locked-in syndrome. Journal of neurology 221: 77-91, 1979. 
7. Uchino A, Sawada A, Takase Y, Kudo S. Symmetrical lesions of the middle cerebellar peduncle: MR imaging and differential diagnosis. Magnetic resonance in medical sciences: MRMS: an official journal of Japan Society of Magnetic Resonance in Medicine 3: 133-140, 2004.

8. Shen Y, Jian W, Li J, Dai T, Bao B, Nie H. Bilateral wallerian degeneration of the middle cerebellar peduncles secondary to pontine infarction: A case series. Journal of the neurological sciences 388: 182-185, 2018.

9. Holmes MD, Brant-Zawadzki MM, Simon RP. Clinical features of meningovascular syphilis. Neurology 34: 553-556, 1984.

10. Brightbill TC, Ihmeidan IH, Post MJ, Berger JR, Katz DA. Neurosyphilis in HIV-positive and HIV-negative patients: neuroimaging findings. AJNR American journal of neuroradiology 16: 703-711, 1995.

11. Feng W, Caplan M, Matheus MG, Papamitsakis NI. Meningovascular syphilis with fatal vertebrobasilar occlusion. The American journal of the medical sciences 338: 169-171, 2009.

12. Vartdal F, Vandvik B, Michaelsen TE, Loe K, Norrby E. Neurosyphilis: intrathecal synthesis of oligoclonal antibodies to Treponema pallidum. Annals of neurology 11: 35-40, 1982.

13. Prange HW, Moskophidis M, Schipper HI, Müller F. Relationship between neurological features and intrathecal synthesis of IgG antibodies to Treponema pallidum in untreated and treated human neurosyphilis. Journal of neurology 230: 241-252, 1983.

14. Link $\mathrm{H}$, Tibbling G. Principles of albumin and $\operatorname{IgG}$ analyses in neurological disorders. III. Evaluation of IgG synthesis within the central nervous system in multiple sclerosis. Scandinavian journal of clinical and laboratory investigation 37: 397-401, 1977.

15. Jones HD, Urquhart N, Mathias RG, Banerjee SN. An evaluation of oligoclonal banding and CSF IgG index in the diagnosis of neurosyphilis. Sexually transmitted diseases 17: 75-79, 1990.

16. Peng $\mathrm{F}, \mathrm{Hu} \mathrm{X}$, Zhong $\mathrm{X}$, et al. $\mathrm{CT}$ and MR findings in HIVnegative neurosyphilis. European journal of radiology 66: 1-6, 2008.

17. Johns DR, Tierney M, Parker SW. Pure motor hemiplegia due to meningovascular neurosyphilis. Archives of neurology 44: 1062$1065,1987$.

18. Gállego J, Soriano G, Zubieta JL, Delgado G, Villanueva JA Magnetic resonance angiography in meningovascular syphilis. Neuroradiology 36: 208-209, 1994.

19. Han JH, Lee CC, Crupi RS. Meningovascular syphilis and improvement with tissue-plasminogen activator (T-PA). The American journal of emergency medicine 22: 426-427, 2004.

20. Umashankar G, Gupta V, Harik SI. Acute bilateral inferior cerebellar infarction in a patient with neurosyphilis. Archives of neurology 61: 953-956, 2004.

21. Flint AC, Liberato BB, Anziska Y, Schantz-Dunn J, Wright CB. Meningovascular syphilis as a cause of basilar artery stenosis. Neurology 64: 391-392, 2005.

22. Asdaghi N, Muayqil T, Scozzafava J, Jassal R, Saqqur M, Jeerakathil TJ. The re-emergence in Canada of meningovascular syphilis: 2 patients with headache and stroke. CMAJ: Canadian Medical Association journal = journal de l'Association medicale canadienne 176: 1699-1700, 2007.

23. Chen MH, Lin CC, Lin TY, Sung YF. Neuro-syphilis, HIV infection and brainstem infarction. QJM: monthly journal of the Asso- ciation of Physicians 106: 1039-1040, 2013.

24. Khamaysi Z, Bergman R, Telman G, Goldsher D. Clinical and imaging findings in patients with neurosyphilis: a study of a cohort and review of the literature. International journal of dermatology 53: 812-819, 2014

25. Yin-Dan Y, Wen-Ke H, Li-Feng G. Meningovascular syphilis with basilar artery occlusion: case report and literature review. Neurology India 62: 213-214, 2014.

26. Bäuerle J, Zitzmann A, Egger K, Meckel S, Weiller C, Harloff A. The great imitator--still today! A case of meningovascular syphilis affecting the posterior circulation. Journal of stroke and cerebrovascular diseases: the official journal of National Stroke Association 24: e1-e3, 2015.

27. Chen TC, Wang JH, Tsai TC. Repeated episodes of acute stroke as manifestation of neurosyphilis in a well-controlled human immunodeficiency virus-infected patient-Successful treatment with ceftriaxone. Journal of the Formosan Medical Association = Taiwan yi zhi 116: 725-726, 2017.

28. Shi M, Zhou Y, Li Y, et al. Young male with syphilitic cerebral arteritis presents with signs of acute progressive stroke: A case report. Medicine 98: e18147, 2019.

29. Plum F, Posner JB. The diagnosis of stupor and coma. Davis, Philadelphia, 1966.

30. McCusker EA, Rudick RA, Honch GW, Griggs RC. Recovery from the 'locked-in' syndrome. Archives of neurology 39: 145-v7, 1982.

31. Sparaco M, Ciolli L, Zini A. Posterior circulation ischaemic stroke-a review part I: anatomy, aetiology and clinical presentations. Neurological sciences: official journal of the Italian Neurological Society and of the Italian Society of Clinical Neurophysiology 40: 1995-2006, 2019.

32. Haig AJ, Katz RT, Sahgal V. Mortality and complications of the locked-in syndrome. Archives of physical medicine and rehabilitation 68: 24-27, 1987.

33. Workowski KA, Bolan GA. Sexually transmitted diseases treatment guidelines, 2015. MMWR Recommendations and reports: Morbidity and mortality weekly report Recommendations and reports 64: 1-137, 2015.

34. Kingston M, French P, Higgins S, et al. UK national guidelines on the management of syphilis 2015. International journal of STD \& AIDS 27: 421-446, 2016.

35. Janier M, Unemo M, Dupin N, Tiplica GS, Patel R. 2014 European guideline on the management of syphilis: giving evidence priority. Journal of the European Academy of Dermatology and Venereology: JEADV 30: e78-e79, 2016.

36. Mageau A, Rodriguez-Régent C, Dion J, et al. Treatment of Syphilis-Associated Cerebral Vasculitis: Reappearance of an Old Question. The American journal of medicine 131: 1516-1519, 2018.

37. Carod Artal FJ. Clinical management of infectious cerebral vasculitides. Expert review of neurotherapeutics 16: 205-221, 2016.

The Internal Medicine is an Open Access journal distributed under the Creative Commons Attribution-NonCommercial-NoDerivatives 4.0 International License. To view the details of this license, please visit (https://creativecommons.org/licenses/ by-nc-nd/4.0/).

(C) The Japanese Society of Internal Medicine Intern Med Advance Publication 